\title{
Mit Sales Development den Verkauf professionalisieren
}

Unternehmen professionalisieren zunehmen ihren Vertrieb länder- und spartenübergreifend. Dazu richten sie zentrale Stellen für Sales Development ein. Aufgabe der Verantwortlichen ist es, den Verkauf zu professionalisieren und zu unterstützen. Allerdings haben Eingriffe im Vertrieb auch eine kritische Komponente: Sie sollen das lokale Engagement nicht schmälern. Es geht um die richtigen Eingriffe, sonst wirkt das Ganze kontraproduktiv.

Christian Belz 
Viele Unternehmen verstärken aktuell ihre zentralen Initiativen im Verkauf. Verantwortliche für Sales Excellence, Sales Enabler oder Sales and Business Development werden ernannt. Zum Beispiel in Unternehmen wie ABB, AXA Winterthur, BASF, Celesio, Gallus, Geberit, Jenoptik, Lienhard Office Group, Medela, SBB Cargo, Schott, Siemens oder ThyssenKrupp. Diese neue Form der Spezialisierung ist nicht nur den großen Konzernen vorbehalten, sondern wird auch von mittleren Unternehmen aufgegriffen. Die Stellen wurden erst vor wenigen Jahren und teilweise ganz neu eingerichtet.

Aufgabe der Verkaufsentwickler ist es, den Verkauf im Unternehmen länderund spartenübergreifend zu professionalisieren und zu standardisieren. In vielen Unternehmen wuchs der Vertrieb dezentral, jede Einheit geht spezifisch vor und die schlechtesten und besten Einheiten unterscheiden sich enorm. Deshalb vertrauen Unternehmen nicht nur auf die lokalen Verbesserungen ihrer Verkäufer im Kontakt mit ihren besonderen Kunden. Sie suchen nach gemeinsamen Lösungen, um Effektivität und Effizienz im Verkauf zu steigern. Oft ist Sales Development international ausgerichtet, muss es aber nicht.

Auch die Verkaufsmanager für Sparten oder Manager für Regionen erweitern ihre Aufgaben. Sie führen nicht nur ihre Verkaufsmannschaft, sondern nutzen Spezialisten. Diese neueren Einheiten in den Unternehmen sind meist intern gut positioniert und arbeiten direkt mit der Geschäfts- und Verkaufsleitung zusammen. Die Teams für Sales Development sind aber klein und sie entscheiden wenig selbst. Sie werden erstaunlich oft durch jüngere Betriebswirte geführt, die eher Fähigkeiten für Konzepte und Projektmanagement einbringen, als praktische Verkaufserfahrung. Die kleinen Teams moderieren den Fortschritt und gewinnen die Verkaufsmannschaften statt zu befehlen. Wichtig ist dabei aber nicht die Form der Organisation, sondern die Aufgabe. Sie lässt sich durchaus auch von Linienvorgesetzten mit geeigneten Projektgruppen erfüllen. Oft werden solche Initiativen auch durch externe Verkaufsberater, Verkaufstrainer oder auf das Verkaufsmanagement spezialisierte Universitäten begleitet.

\section{Gefahr durch Orientierungslosigkeit}

Die Idee eines zentralen Sales Development ist plausibel. Weil der Erfolg der meisten Unternehmen durch den Verkauf getrieben wird, lohnt es sich auch hier anzusetzen. Was soll aber ein Verantwortlicher für Sales Development tun, um den Anspruch für mehr Professionalisierung und Wirksamkeit zu erfüllen? Welche Verkaufsthemen, Projekte oder Change-Prozesse sind im Zeitablauf ergiebig? Wie wird ein zentraler Spezialist von vielen Verkaufsverantwortlichen akzeptiert und wie muss er sich im Unternehmen vernetzen? Was sind die Erfolgsausweise für eine solche Aufgabe? Manche ernannten Sales Developer scheinen dabei etwas orientierungslos, ebenso wie ihre Vorgesetzten. Die Gefahr ist recht groß, dass sich ihre Aufgabe schrittweise von der großen Idee entfernt. Rasch sind sie dann nur noch die Erfüllungsgehilfen für Nebenaufgaben im Verkaufsmanagement.

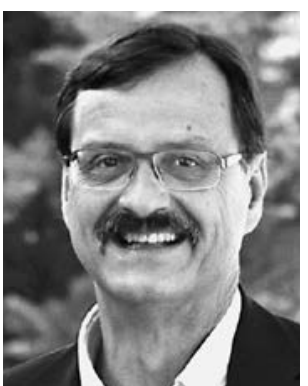

Prof. Dr. Christian Belz

ist Ordinarius für Marketing an der Universität St. Gallen und Direktor am Institut für Marketing.

Christian Belz

Universität St. Gallen, St. Gallen, Schweiz E-Mail: christian.belz@unisg.ch 
Verkäufer ergreifen ihren Beruf meistens, weil sie selbstständig sein wollen. Ihre Wege zum Erfolg sind sehr verschieden. Sie versuchen sich gegenüber einer Zentrale mit Eingriffen im Verkauf abzuschirmen oder wehren sich dagegen. Unternehmen stellen demgegenüber oft fest, dass die Verkäufer nicht genügend professionell und konform zur Strategie vorgehen. Deshalb erwägen sie, den Verkauf zentral zu beeinflussen.

Die Gestaltung eines zentralen Sales Development sollte behutsam erfolgen. Eingriffe sollen die Motivation der Verkäufer nicht schmälern, sondern ihre Kraft freisetzen. Auch sollte Verkäufern ein großer Freiraum zugestanden werden. Manche Eingriffe sind zwar fein ausgedacht und gut gemeint, bleiben aber illusionär. Jeder zentrale Verkaufsentwickler wird daher sorgfältig abschätzen, was er mit seinen Ressourcen beeinflussen kann. Es lohnt sich nicht, Dinge nur anzustoßen oder lediglich Unruhe zu stiften.

Ohne Zweifel ist es wertvoll, die Sicht der Verkäufer ernst zu nehmen. Viele fühlen sich durch Zentralen eher behindert als unterstützt. Es lohnt sich zudem, zu untersuchen, wie solche zentralen Sales Developer in der Praxis vorgehen und wie das im Verkauf ankommt. In einer ersten Phase 2012 fand am Institut für Marketing der Universität St. Gallen eine Diskussion mit 18 Verantwortlichen für den internationalen Vertrieb von Unternehmen darüber statt, wie sie eingreifen, um den internationalen Vertrieb zu professionalisieren. Die Ergebnisse wurden unter dem Titel "Global Sale“ zusammengefasst. Gemeinsam mit Mercuri International Deutschland wird das Institut für Marketingin einer zweiten Phase 2017 erneut intensive Gespräche mit den Experten für Sales Development führen. Geplant ist eine repräsentative Befragung.

\section{Kerngedanken}

1. Verkauf braucht nicht nur Führung, sondern auch Verkaufsentwicklung.

2. Die zehn Eingriffe im Vertrieb betreffen strategische Integration, Verkaufsleistung, Verkaufs-KPIs, Struktur und Prozesse, CRM und Verkaufsmethoden, Verkaufsdialog „nahe an der Front“, Nutzung internen Know-hows, Global Account Management, Qualifikation der Vertriebsmitarbeiter und Zusammenarbeit von Marketing und Verkauf.

3. Verkaufsentwicklung braucht eine ausgewogene „Roadmap“.

\section{Hebel zur Professionalisierung des Verkaufs}

Es lohnt sich, die möglichen Ansätze der Verkaufsentwicklung grundsätzlicher und systematisch anzugehen. Dabei wird grundsätzlich zwischen zwei Aufgaben zur Professionalisierung des Vertriebs unterschieden:

1. Sales Development: Es geht darum zu analysieren, zu konzipieren, zu projektieren und den Verkauf methodisch zu stützen. Zusammengefasst werden die Ausrichtung, Inhalte und interne sowie externe Zusammenarbeit im Vertrieb vorbereitet.

2. Verkaufsführung: Erst die aktive Verkaufsführung kann jedoch aufgreifen, umsetzen, führen, unterstützen und kontrollieren. Verkaufsführung erfordert auch eine starke Machtposition des Verantwortlichen im Unternehmen.

Während den ersten Bereich eher die jungen Sales Developer wahrnehmen, ist der zweite Ansatz besonders die Domäne der Vertriebsleiter mit ihren großen Mannschaften von Verkäufern. Die Arbeitsteilung ist jedoch nicht fest, denn der Sales Developer kann auch aktive Führungsaufgaben übernehmen, während sich der Vertriebsleiter auch im Sales Development engagiert. Der Vertriebsleiter entscheidet zumindest, welche Neuerungen im Vertrieb schließlich aufgegriffen werden. Eine enge Zusammenarbeit ist in jedem Fall eine wichtige Voraussetzung für den Erfolg.

Inhaltlich können sich Sales Development und Führung auf nahezu sämtliche Themen im Verkauf richten. Das reicht zum Beispiel von Zukunftsszenarien für den Vertrieb, Digitalisierung im Verkauf, Targeting von Kunden, Value Selling, Zusammenarbeitsmodellen zwischen Vertrieb, Marketing,Technik, Produktmanagement, Kundendienst und Controlling über Verkaufsintegration im Multichannel-Vertrieb, Coaching der Vertriebsmitarbeiter und KPIs im Vertrieb bis zu Vertriebsinnovation und -kreativität.

Übergreifend wurden die Hebel zur Professionalisierung des Verkaufs sowohl für das Sales Development als auch die Verkaufsführung ermittelt (siehe Abbildung 1).

Es kann nicht Ziel des Sales Development und der Verkaufsführung sein, sämtliche Hebel zu gewichten. In der Praxis treffen wir typische Muster an, wie sie die Abbildung mit Ausprägungen darstellt. Tatsächlich gibt es eher Analysten und Konzeptionisten in der Zentrale. Sie führen stark top-down und setzen besonders auf Alignment von Strategie und Verkauf, Performance, Metrics und Methoden. Eine andere Gruppe konzentriert sich konsequent auf die Verkaufsfront und gewichtet einen starken Dialog (close to people) mit den 
Verkäufern. Sie befasst sich intensiv mit der Qualifikation der Verkaufsmitarbeiter; erst qualifizierte Personen können selbstständig und unternehmerisch wirken.

Schließlich konzentrieren sich manche Unternehmen vorwiegend auf organisatorische Maßnahmen und bewirken durch Reorganisationen und neue Schwerpunkte in den Ressourcen eine Erneuerung des Verkaufs. Beispielsweise gruppieren sie die Länderverantwortung neu, stellen sich neu nach Segmenten auf und degradieren damit frühere Länderfürsten oder sie verlagern ihre Ressourcen auf Schlüsselmärkte. Ebenso werden in der Zentrale Global Accounts angesiedelt, die für eine länderübergreifende Zusammenarbeit mit Kunden in mehreren Ländern oder überregional zuständig sind. Diese Beispiele verdeutlichen: Es gilt, die Eingriffe richtig zu koordinieren und zu gewichten. Inhaltliche und menschliche Anforderungen an die Verantwortlichen sind dabei je nach Eingriff sehr verschieden.

Die Eingriffe zur Professionalisierung richten sich je nach Unternehmen auf Tochtergesellschaften, Vertriebsregionen,
Verkauf von Sparten oder Produktbereichen, Großhändler und Einzelhändler sowie Vertretungen. Gemeinsam ist dabei, dass mit bisher recht autonomen Einheiten eine stärkere, gemeinsame Professionalisierung des Verkaufs verfolgt wird.

\section{Hebel für Eingriffe im Verkauf}

Die einzelnen Hebel für Eingriffe im Verkauf seien hier in Kürze erläutert:

1. Alignment - Unternehmens- und Verkaufsstrategie abstimmen: Ziel des Alignments ist es, die Unternehmensstrategie auch verkaufsbezogen auszurichten, das Know-how und die Bedingungen des Verkaufs zu nutzen, die Strategie für den Verkauf zu konkretisieren, die Akzeptanz der Strategie im Verkauf zu sichern und das Engagement für eine Umsetzung zu steigern. Manche Strategien wirken nämlich abgehoben und oberflächlich. Inhaltlich gilt es, die Stoßrichtung von Leistungen, Kunden sowie Kanälen und die Bedingungen der Organisation in den Verkauf zu übersetzen.

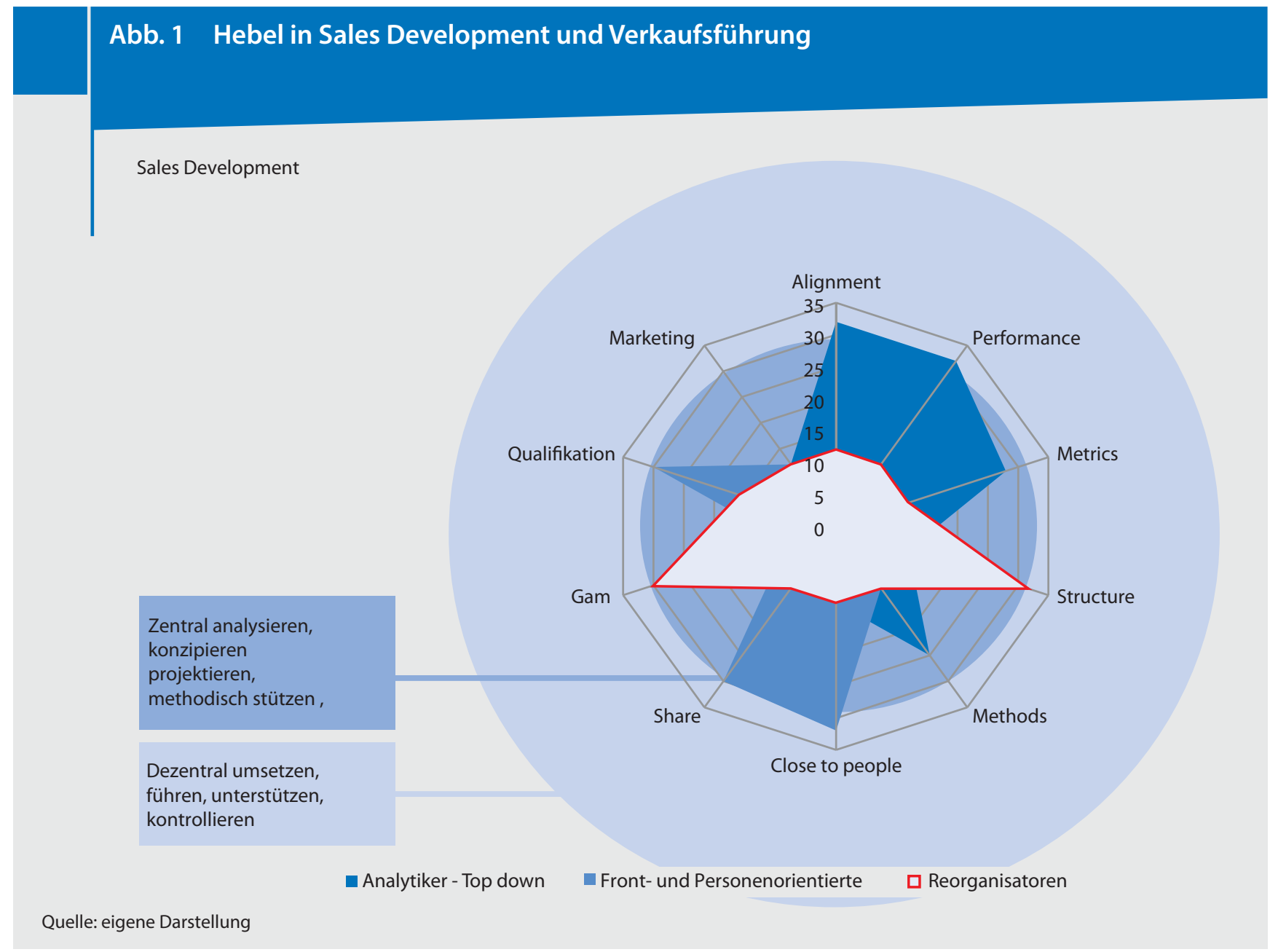


2. Performance - Sales Excellence im Unternehmen definieren: Ziel des Performance-Ansatzes ist es, die Leistung des Vertriebs systematisch und umfassend für das Unternehmen zu spezifizieren und nach Ländern bis zu einzelnen Vertriebsmitarbeitern selektiv zu verbessern. So können beispielsweise für ein Land oder eine Vertriebseinheit von 15 Kriterien zur Performance nur die Kriterien 8 und 12 besonders wichtig sein. Die definierte Performance ist die Grundlage für intensive Diskussionen um Qualifikation und Ergebnisse. Inhaltlich ist dieser Ansatz vielfältig und verbindet auch mit weiteren Hebeln sowie Maßnahmen.

Die Systematik unterscheidet von jenen Unternehmen, die für jede Vertriebseinheit nur sehr situativ und oft auch reaktiv vorgehen. Leicht springen zentrale Verantwortliche nur noch von einer zur nächsten „Feuersbrunst“ in den Niederlassungen und werden bereits durch negative Vorkommnisse ausgelastet.

3. Metrics - Sales Analytics, KPI's und Controlling bestimmen: Mit dem Ansatz der Metrics wollen Unternehmen die Leistung und Ergebnisse des Vertriebs messen und damit besser steuern. Messung liegt im Trend und Controlling erlaubt einen raschen Zugang zur Leistung und zu den Vertriebseinheiten über Distanz. Manche Unternehmen betonen diesen Zugang besonders, weil sie die Vertriebseinheiten durch den Controller führen lassen, natürlich im Zusammenspiel mit der Geschäftsführung. Der Controller hat jedoch einen sehr einseitigen Zugang zur Vertriebsprofessionalisierung. Metrics ersetzen keine starke Führung.

Während der Performance-Ansatz qualitative und quantitative Größen berücksichtigt, stehen hier Kennzahlen oder Key Performance Indicators (KPIs) im Vordergrund. Beispiele sind Umsätze nach Kunden und Leistungen, Anteile neuer Produkte und Services, Umfang

\section{Zusammenfassung}

Unternehmen richten vermehrt zentrale Stellen für Sales Development ein. Ihre Aufgabe ist es, den Verkauf überregional und spartenübergreifend zu professionalisieren. Aus zehn häufigen Eingriffen im Vertrieb gilt es zu wählen und umzusetzen, was für das eigene Unternehmen passt. der Angebote (oder Preisqualität), Erträge oder Besuchszahlen. Besonders im Projektgeschäft spielt die Nachkalkulation der erfüllten Aufträge eine Rolle.

Das Management stützt sich in der Regel auf ein Cockpit mit den Kennzahlen zu den einzelnen Vertriebseinheiten. Dabei ist es gefährlich, nur die negativen Ausreißer mit Ursachen und Maßnahmen zu gewichten. Ebenso wichtig ist es, die besonders erfolgreichen Einheiten noch mehr zu fördern.

Sales Metrics sind in Unternehmen auch die Basis, um die variablen Lohnanteile im Vertrieb zu bestimmen. Dabei werden meist Individual- und Unternehmensbonus berücksichtigt. Auch kann es eine Rolle spielen, die Lohnsysteme der Unternehmenseinheiten maßvoll zu harmonisieren. Maßvoll, weil beispielsweise die Unterschiede zwischen asiatischen, amerikanischen und europäischen Ländern oft recht groß bleiben müssen.

Immer prägen Kennzahlen und Auswertungen auch die Aufmerksamkeit des Vertriebs. Im positiven Fall regen sie die Diskussion um die Ursachen der besten Vertriebsergebnisse im Unternehmen an und motivieren alle Einheiten, sich zu verbessern. Die Zusammenhänge mit dem Hebel „Share“ sind offensichtlich.

4. Structure - Verkauf organisieren: Ziel von organisatorischen Eingriffen ist es, die Strukturen und personellen Ressourcen auf die Strategie auszurichten und die Zusammenarbeit von zentralen Abteilungen sowie Spezialisten mit dezentralen Vertriebsregionen zu erleichtern und zu sichern.

Dabei gilt es, eine einfache Erkenntnis zu beachten: Setzt ein Unternehmen große zentrale Ressourcen für die Verkaufsentwicklung ein und hält die Vertriebseinheiten schlank, so funktioniert das Zusammenspiel - ebenso wie umgekehrt. Erst bei je größeren zentralen und dezentralen Ressourcen beschäftigen sich die Verantwortlichen gegenseitig und Konflikte werden verstärkt. Es lohnt sich deshalb sorgfältig zu prüfen, welche Funktionen zentral und dezentral angesiedelt werden.

In manchen Unternehmen werden die Regionen neu zusammengefasst und teilweise neue Hierarchiestufen eingezogen. Es spielt beispielsweise eine Rolle, ob Deutschland, Österreich und die Schweiz eigenständig operieren und direkt dem Topmanagement berichten oder ob sie in einer DACH-Region unter einer Leitung zusammengefasst sind. Auch lassen sich die Aufgaben und Kompetenzen jeder Stufe verschieden ausgestalten. 
Besonders in Unternehmen mit einer Matrixorganisation befassen sich die Sparten oder Business Units damit, wie sie einen Vertrieb organisieren und unterstützen können, der auch weitere Sparten und Business Units verkauft. Ziel ist es, mehr Aufmerksamkeit und Engagement des Vertriebs für diesen Bereich in den Niederlassungen zu mobilisieren, als es dem gegenwärtigen Umsatz entspricht. Gleichsam wird versucht, 40 Prozent Einsatz für 20 Prozent Umsatz zu bewirken. Dann sind die Voraussetzungen für ein Wachstum gegeben.

In manchen Unternehmen ist die Struktur von Niederlassungen recht zufällig gewachsen und jede Einheit organisiert sich verschieden. Hier helfen „Blueprints“ für die angestrebte Organisation von Niederlassungen. Nötige Abweichungen werden intensiv diskutiert.

Organisatorisch spielt auch eine Rolle, ob ein Unternehmen den direkten oder indirekten Vertrieb wählt. Indirekter Vertrieb ist für kleinere Märkte oder spezifische Kundenzugänge geeignet. Grundsätzlich können aber eine Tochtergesellschaft und eine Vertretung die Strategie eines Unternehmens gleich gut oder schlecht umsetzen. Manchmal verselbstständigen sich auch Vertretungen, weil sie nicht professionell betreut und unterstützt werden.

5. Methods - Customer Relationship Management und Prozesse zu Kundengewinnung: Eingriffe in diesem Bereich sollen das Vorgehen im Verkauf vereinheitlichen und professionalisieren. Methoden sind dabei vielfältig. Sie lassen sich aber besser standardisieren als Inhalte. Umfassend sind zum Beispiel die vielen Initiativen in Unternehmen, um ein weltweites Customer Relationship Management zu installieren. Dabei werden oft viele Teillösungen in den Einheiten abgelöst. Kombiniert werden diese Ansätze beispielsweise mit Tablets, die mit den richtigen Informationen bespielt sind und sich in der Interaktion mit Kunden wirksam nutzen lassen. Auch Methoden zur zuverlässigen Erfassung der Marktpotenziale, zu Sales Funnels bis zu strukturierten Verkaufsgesprächen sind in der Methodenbank anzutreffen.

Bei neuen Methoden für das gesamte Unternehmen werden besonders die besten Einheiten und Verkäufer bestraft. Die zentralen Lösungen hinken ihren Ansätzen nach, sie werden im Vergleich zu eingespielten, eigenen Ansätzen als schlechter empfunden. Eine Umstellung führt damit zu mehr Aufwand und offenbar steigt nur der zentrale Nutzen einer Standardisierung, nicht aber der dezentrale. Zentralen tun gut daran wichtige Themen aufzugreifen, die noch brachliegen oder mit neuen Lösungen auch wesentliche Fortschritte für alle Betroffenen zu erreichen und zu kommunizieren.

6. Close to people - Permanenter Dialog mit Verkaufsverantwortlichen: Ziel dieses Ansatzes ist es, die wichtigen Veränderungen im Verkauf vor Ort mit den Verantwortlichen zu erörtern und Fortschritte konkret zu begleiten. Oft ist das Vorgehen eher dezentral und spezifisch, also wenig standardisiert. Die aktuellen Probleme in jedem Bereich stehen im Vordergrund. Der Führungsanteil ist weit stärker als der Entwicklungsanteil.

Um nötige Maßnahmen vor Ort zu diskutieren, reisen die Verantwortlichen viel. Sie stellen sich den vielfältigen Herausforderungen in den Einheiten, setzen Ziele, entwickeln spezifische Maßnahmen und verfolgen die Realisierung. Und: Sie festigen intern die persönlichen Beziehungen zu den Niederlassungen und begleiten zu wichtigen Kunden. Typisch die Aussage eines zentralen Verkaufsentwicklers: „Ich saß letztes Jahr 35 Tage nur im Flugzeug und bewegte mich 150 Tage im Ausland."

Weil diese Verantwortlichen laufend unterwegs sind, haben sie eher das Problem, sich in der Zentrale richtig zu positionieren. Weil sie immer weg sind, fehlt die Präsenz in den Gremien, Sitzungen und Workshops der Zentrale.

Um viele Niederlassungen konkret vor Ort zu begleiten, braucht es Ressourcen. Oft sind in der Zentrale nur eine Handvoll Leute für 40 bis 50 Länder zuständig, was den möglichen Einfluss begrenzt.

7. Share - Know-how im Verkauf teilen: In vielen Unternehmen existieren ausgezeichnete Erfolgsbeispiele mit Kunden oder ausgereifte Marketing- und Verkaufsmaßnahmen. Ziel dieses Ansatzes ist es, die dezentralen Er-

\section{Handlungsempfehlungen}

- Bestimmen Sie die Agenda für einen professionellen oder exzellenten Vertrieb.

- Lancieren Sie gezielte Projekte für die wichtigen Verkaufsthemen.

- Verbinden Sie Verkaufsführung und Verkaufsentwicklung.

- Gewinnen Sie Vertrauen und Akzeptanz des Vertriebs für angestrebte Neuerung. 
folge auch für weitere Einheiten im Unternehmen zu nutzen. Gleichsam werden gute und auch zufällige Lösungen „bottom-up“ multipliziert und damit gestärkt. Funktionierende Beispiele der Kollegen wirken besonders glaubwürdig für die Beteiligten.

Know-how im Vertrieb zu teilen ist anspruchsvoll. Lösungen sind vielfältig und in jeweils besonderen Konstellationen entstanden. Es gilt kritisch zu prüfen, was übertragbar ist. Manche Einheiten fühlen sich als interne Konkurrenten oder wollen ihren Kollegen nur freiwillig helfen. Flankiert wird auch dieser Austausch durch die omnipräsente Überlastung, besonders der Besten.

Während „Share" eher vorhandene und erfolgreiche Lösungen aufnimmt, ist es auch möglich, wichtige Ansätze zu initiieren. Mit lancierten Pilotproketen und Experimenten in den Vertriebseinheiten lassen sich analoge Fortschritte erzielen. Know-how wird in einer offenen Kultur von Unternehmen geteilt, in welcher die gegenseitige Akzeptanz und Wertschätzung groß sind.

8. GAM - Globale Kunden zentral bearbeiten: Internationale Anbieter arbeiten mit internationalen Kunden zusammen. Ziel ist es, für den Kunden weltweit qualifizierte Leistungen (nach definierten Standards) zu bieten. Naturgemäß gelingt das nur, wenn der Kunde nicht nur länderbezogen bewertet und bearbeitet wird. Trotzdem spielt das Zusammenspiel zwischen einem zentralen Account Management und den dezentralen Einheiten eine wichtige Rolle. Zentrale Abmachungen müssen mit der eigenen Organisation dezentral für die Einheiten des Kunden erfüllt werden.

Die Interessen von globalem Key Account Management und Vertriebseinheiten entsprechen sich oft nicht. Es geht dabei häufig um Diskussionen der Zuordnung von Margen sowie der Preise. Der globale Kunde Nummer 3 kann in einem Land vielleicht erst Nummer 18 sein. Damit stehen globales und regionales Konditionengefüge im Wettstreit.

Auch ein einzelnes Land kann mit verschiedenen Vertriebsgebieten bearbeitet werden. Ebenso können die Kunden mit Standorten in mehreren Gebieten ganzheitlich bearbeitet und die laufende Zusammenarbeit optimiert werden. Probleme und Lösungen sind analog zum Global Account Management, wenn auch weniger komplex.

9. Qualifikation - Verkäufer qualifizieren: Die Zahl der Verkaufsmitarbeiter ist in den meisten Unternehmen sehr groß. Verkaufsmanagement ist ein „people business“. Ziel ist es, mit diesen Eingriffen die bestehenden Verkäufer kritisch zu prüfen. Ein Schema der Sales Performance ist dazu hilfreich. Auf dieser Basis lässt sich bestimmen, welche bestehenden Verkäufer den Anforderungen nicht genügen. Bei einschneidenden Anpassungen, einer Expansion oder auch bei einer größeren Fluktuation der Mitarbeiter im Verkauf wird die Neubesetzung von Verkäuferstellen besonders wichtig. Es gilt, die Verkäufer mit Potenzial anzuziehen, zu selektionieren, einzuführen und zu entwickeln. Es genügt nicht, Verkaufstalente einzustellen. Erst danach entscheidet sich, ob es dem Unternehmen gelingt, diese Talente auch zu nutzen.

Bei der persönlichen Qualifikation der Mitglieder einer Verkaufsmannschaft spielt das Training der Verkaufsmitarbeiter (für alle aufgezeigten Hebel) meist eine zentrale Rolle. Tatsächlich lancieren manche Unternehmen zentrale Programme zur Weiterbildung des Vertriebs für das gesamte Unternehmen, selbst wenn sie keine zentralen Stellen für eine starke Verkaufsführung und Sales Development haben. Die Wirksamkeit von Schulungen hängt aber eng mit der Führungsstärke zusammen. Sonst bleibt der Lerntransfer das Problem.

10. Marketing - Verkauf unterstützen und Leads qualifizieren: Ziel dieses Ansatzes ist es, den Kunden in enger Zusammenarbeit zwischen Marketing und Vertrieb zum Kauf zu führen. Wichtig ist dabei das Lead Management.

Detaillierte Analysen der Kaufschritte von Kunden und Nichtkunden zeigen, wo mögliche Hebel liegen, um mit Marketing und Vertrieb zu fördern. Vielfältige Instrumente und Kanäle gilt es auf den Kaufprozess des Kunden auszurichten. Das Marketing qualifiziert die Kundenleads für den Verkauf. Verbreitet ist dabei der Konflikt, dass Marketing die Kunden nur wenige Schritte in ihrem Interesse bewegt und der Verkauf die Anstrengungen nicht fortsetzt, weil er Hinweise zu Kunden verlangt, die bereits vor dem Abschluss stehen. Diese Lücke gilt es zu überbrücken.

Manche der früher aufgezeigten Aufgaben für Sales Development werden in Unternehmen auch dem zentralen Marketing delegiert. Beispielsweise gehören Aufgaben zum Customer Relationship Management oder Methoden zur Ermittlung der Marktpotenziale dazu. Diese Zuordnung funktioniert nur, wenn das Marketing sehr nahe am Verkauf und an den Kunden vorgeht.

Relevant ist für alle gewählten Hebel, wie rasch und verbindlich die neuen Anforderungen im Verkauf umgesetzt werden. 
Manche Unternehmen diskutieren lange, suchen nach Kompromissen, warten ab oder nehmen viele Zwischenschritte in Kauf. Dieses Vorgehen ist nur vermeintlich menschlicher, die schwelende Unklarheit belastet die Mitarbeitenden stärker.

\section{Erhebliche Widerstände einkalkulieren}

Es lässt sich keine allgemeingültige Roadmap für Sales Development entwickeln. Es handelt sich eher um eine Initiative mit offenen Zwischenschritten, wobei die Bedingungen von Markt und Unternehmen das Vorgehen prägen. Auch sind die Möglichkeiten bei eigenen Tochtergesellschaft und Vertriebseinheiten oder Sparten anders als bei Händlern und Vertretungen.

Einige allgemeingültige Schritte beim Aufbau eines Sales Developments lassen sich jedoch festhalten:

1. Verkaufsagenda: Es gilt, die relevante Verkaufsagenda für das Unternehmen zu bestimmen. Die Ziele, der Fokus und Etappen begründen den Aufbau eines Sales Development. Wichtig ist es festzulegen, was zuerst und was später aufgegriffen wird. Mit gewissen Themen lassen sich für das Sales Development auch leichter Erfolge erreichen und belegen.

2. Entwicklungsprojekte: Gestützt auf die gewählten Themen werden Projekte mit Zielen, Zwischenschritten, Teilthemen, Teams und Kosten definiert und geführt.

3. Integration in der Führung: Neue Lösungen werden in die Führung integriert. Für die Umsetzung ist ein enger Führungsrhythmus wichtig. Für Neuerungen im Vertrieb gilt es zu kämpfen. Viele Menschen auf der Seite des Unternehmens und der Kunden sind beteiligt, manche Neuerungen sind sehr qualitativ geprägt und eine große Zahl von Transaktionen wird gestaltet.

4. Kommunikation: Neuerungen im Verkauf brauchen Kommunikation, Akzeptanz und Vertrauen, Vertiefung, Training und Coaching.

Ein Sales Development einzurichten ist nicht trivial. Teilweise verändert sich damit die Kultur eines Unternehmens. Die unternehmerischen Freiräume des Vertriebs werden eingeschränkt. Damit werden auch indirekt die Probleme des Sales Developments angeschnitten. Die Widerstände sind oft erheblich. Ein Veränderungsbedarf wird verneint, vermeintlich haben die Vertriebseinheiten alles schon bestens im Griff.

Die Akzeptanz zu gewinnen fordert die Verkaufsentwickler permanent heraus. Sie brauchen das spürbare Engagement des Managements und die klare Anbindung an die Vertriebsführung. Hilfreich ist es auch, die Vertriebseinheiten als interne Kunden zu behandeln. Mit dieser Haltung lassen sich manche Konflikte zwischen Zentrale und Niederlassungen vermindern oder produktiv nutzen. Wesentliche Treiber für Erfolge sind gemeinsame Interessen von Zentrale und Vertriebseinheiten. Die Einheiten wollen durch zentrale Initiativen gewinnen und nicht verlieren.

Zwischen Zentrale und Vertriebseinheiten muss ein Schwungrad entstehen. Themen mit beiderseitigem Vorteil aufzugreifen ist dabei entscheidend.

\section{Quellen}

Belz, Ch. (2016): Customer Journey - Qualifikation für Kundenprozesse, in: Marketing Review St. Gallen, Nr. 3, S. 60-67, St. Gallen

Belz, Ch. (2016): Lead Management, in: Marke 41, Nr. 4, S. 8-13, St. Gallen

Belz, Ch. (2016): Sales Heatmap I und II, in: Swiss Marketing Review, Nr. 3, S. 12-18, Nr. 4, S. 18-25, St. Gallen

Belz, Ch., Betz, M. (2015): Know-how im Vertrieb teilen, in: Marke 41, Nr. 4, S. 8-13, St. Gallen

Belz, Ch., Bussmann, W., Lee, Y.-Ch. (2013): Global Sales - zentrale Eingriffe im internationalen Vertrieb, in: Marke 41, Nr. 5, S. 8-15, St. Gallen

专 Schmitz, Ch. (2006): Internationales Vertriebsmanagement für Industriegüter - Handlungsimplikationen aus dem Blickwinkel internationaler Tochtergesellschaften und Vertretungen, Wiesbaden (www.springerprofessional.de/link/4596574)

르 Abonnenten des Portals www.springerprofessional.de erhalten diesen Beitrag kostenlos im Volltext.

Weitere Empfehlungen der Verlagsredaktion aus
www.springerprofessional.de zu:
Q Business Development
Kohne, A.: Business Development, in: Kohne, A.: Business
Development, Wiesbaden, 2016, www.springerprofessional.
de/link/10499530
Kohne, A.: In sechs Schritten zum Business Development, in:
Kohne, A.: Business Development, Wiesbaden 2016, www.
springerprofessional.de/link/10499542
Homburg, Ch.: Internationales Marketing, in: Homburg, Ch.:
Marketingmanagement, Wiesbaden 2017, www.springer-
professional.de/link10736460

